\title{
Adding Synthetic Detail to Natural Terrain Using a Wavelet Approach
}

\author{
Mariano Perez, Marcos Fernandez and Miguel Lozano \\ Department of Computer Science, University of Valencia, \\ Valencia, Spain \\ \{Mariano.Perez, Marcos.Fernandez, Miguel.Lozano\}@uv.es
}

\begin{abstract}
Terrain representation is a basic topic in the field of interactive graphics. The amount of data required for good quality terrain representation offers an important challenge to developers of such systems. For users of these applications the accuracy of geographical data is less important than their natural visual appearance. This makes it possible to mantain a limited geographical data base for the system and to extend it generating synthetic data.

In this paper we combine fractal and wavelet theories to provide extra data which keeps the natural essence of actual information available. The new levels of detail(LOD) for the terrain are obtained applying an inverse Wavelet Transform (WT) to a set of values randomly generated, maintaining statistical properties coherence with original geographical data.
\end{abstract}

\section{Introduction}

Terrain representation is a basic topic in the field of training simulators, in both military and civil applications. It is obvious that, in these representations, the amount of geographic data provided to the subject is clearly related to the feeling of visual immersion achieved by the system. However it, is not so obvious that accuracy of the geographical data is less important than their natural visual appearance. Usually the terrain representation is based on a digital elevation model (DEM) plus a set of textures mapped on the mesh. The viewer integrates this geographic information without realizing about the real source of the data presented but about the natural apparency of the final representation.

Other important topic in the field of simulation and interactive graphics is the freedom of movement within the synthetic environment, that should be as similar as possible to the real world. Additionally, is expected to have an homogeneous visual quality from every possible point of view. This introduces an important drawback in most of the current terrain representation models, which have a limited resolution to shown to the viewer. For instance, a flight simulator offers a good quality appearance of the terrain representation when observed from high altitude. This also allows the coverage of large scale terrains. However, for low height fly mission, the visual quality of the representation suffers an important 
degradation, because the original terrain model is not accurate enough to provide the required detail of the representation.

A possible solution to the previously indicated problem could be to resize the geographical data base by adding extra data for those special purposes. Nevertheless, there will be always some limitations which come mainly from: budget restrictions, availability (it is not always possible to have access to the data with the required accuracy), resources consumption (storage space, computing, etc.). An approach to solve the resize problem could be the addition of extra resolution, to real terrain meshes, by means of the natural generation of new synthetic data. In order to maintain the natural appearance and the fidelity to the original terrain, the extra data should statistically follow the properties extracted from the original data. To accomplish this goal we propose a combination of the fractal (in concrete $1 / f$ processes) and wavelet theory.

Considering the limits in storage capacities, our solution will not store the generated data; on the contrary it will be dynamically generated when needed. This means that generated data has to be compatible with the temporal contrainsts of the simulation system.

\section{Fractal and Multifractal Functions}

The family of $1 / f$ fractals has been successfully used to model a wide variety of natural phenomena and even sociological aspects of the human behavior. Well known examples are: natural landscapes, distribution of a river turbulent flow, evolution of the stocks in the markets, etc. [12]

An important characteristic of the $1 / f$ processes is that its spectral density presents an exponential behavior. The spectral density decades on frequency according to the following equation:

$$
S(f) \sim \frac{1}{f^{\gamma}}
$$

where $f$ is the frequency and $\gamma$ is a constant.

This means that a log-log representation of this density with respect the frequency is an straight line with a slope of $-\gamma$.

Fractional Brownian motion ( $\mathrm{fBm}$ ) is probably the best known mathematical characterization of the $1 / f$ processes [7]. This theory has been frequently studied due to its simplicity and the wide range of natural phenomena that is able to model.

Based on the works from Mandelbrot, is possible to represent fractals objects using "fractal dimension". This parameter has an integer value for non-fractals objects ( 1 for curves, 2 surfaces,etc.), whereas for fractal objects the fractal dimension has a non integer value bigger than its topological dimension. The fractal dimension can be used as a roughness index. For instance, in case of curves, values close to one mean low roughness in the function, and values close to two correspond with extremely rough functions. FBm fractal functions are characterized by having only one fractal dimension with an homogeneous distribution over the whole domain. This type of fractal objects are known as monofractals. 
There are other kinds of fractals which present a variation of the fractal dimension along its domain; this family of fractals is usually referred as multifractals. The Multifractals were introduced firstly to model energy dissipation turbulence's [8][5]. They have been proved to be adequate to model a wide range of non-homogeneous processes [11] [10]. One important consequence of the lack of homegenity in the fractal dimension is that the increments are not stationary, so there is no accurate value for the fractal dimension parameter. It will change in an erratic way along the domain.

\section{Wavelets on the Generation of $\mathrm{fBm}$}

Two of the most interesting properties of the $\mathrm{fBm}$ noise are its non stationary behavior, with stationary increments, and its self-similarity at different scales. The stationarity property implies a time-dependent analysis, while self-similarity requires some scale-dependent analysis. These two characteristics are intrinsic to in the wavelet transform [6] [2], which makes them a powerful tool for the analysis and synthesis of fBm's.

An orthogonal wavelet decomposition of a function $X(t)$ generates detail and coarse coefficients recursively by using the equations:

$$
\begin{aligned}
c_{j, i} & =\sum_{k} c_{j+1, k} h[-i+2 k] \\
d_{j, i} & =\sum_{k} c_{j+1, k} g[-i+2 k]
\end{aligned}
$$

being $c_{j, i}$ and $d_{j, i}$ the coarse and the detail coefficients respectively. Being $h$ and $g$ the low and high filter coefficients associated to the wavelet base.

The statistical behavior of these coefficients was previously analyzed by Faldrin [4]. Based on the results of this studies it is possible to assume in practical applications that the correlation amongst the detail coefficients can be ignorated as Wornell point out in [13]. Therefore, it is possible to consider these coefficients as a set of independent Gaussian variables. As a summary, what Wornell discovered is a quite simple procedure to synthesize nearly-fBm functions. The similarity between the results using this method and a pure fBm is directly related to the number of "vanish moments" of the wavelet base selected. Even though, it is not necessary to use basis with high number of vanish moments. In fact, the only important restriction is to select wavelet bases with the regularity property. [12].

\section{Terrain Representation Based on $\mathrm{fBm}$}

Natural landscapes are examples of self-similar fractal phenomena where the geometrical structure is invariant when examined at different scales. This qualitative 
characterization is the base for most approaches to synthetic terrain generation [3], [1]. The first attempts to the generation of fractal landscapes were made by Mandelbrot several years ago [9]. .

If we consider $V_{H}(x, y)$ a Brownian surface with a Holder parameter $H$ $(0<H<1)$, every section of this surface obtained by a vertical plane, generates a fBm curve with $H$ parameter. Based on this property, if we move a distance $\Delta r=\Delta x^{2}+\Delta y^{2}$ over the surface, the expected value of the function variation will be:

$$
\operatorname{Var}\left(V_{H}\right) \propto \Delta r^{2 H}
$$

The fractal dimension of this surface is bigger than the topological dimension of the corresponding non-fractal surface.

\section{Approaches to Increase the Resolution of a Terrain Mesh}

After the introduction of some basic concepts about fractals functions and wavelets, we will explain our approach, based on these principles, to increase the resolution of a natural terrain mesh by adding new levels of detail (LOD). The new synthetic LODs preserve the statistical behavior intrinsic to the real data.

\subsection{Global Scope Approach (GSA)}

The proposed approach is based on the generation of nearly-fBm functions using the results of the statistical behavior analysis of the detail coefficients in pure fBm [13].

As previously indicated, the $\mathrm{fBm}$ has self-similarity at every scale. If we assume that the terrain meshes are $\mathrm{fBm}$, or at least they are close enough to it, the new LODs generated using this technique maintain the statistical properties of the original natural mesh. The assumption includes that the variance of detail coefficients follows a decreasing power-law.

Taking into account the bidimensional nature of the meshes for the terrain representation of the WT, three different types of coefficients will be generated: horizontal $\left(d_{(h) j,}\right)$, vertical $\left(d_{(v) j}, \vec{k}\right)$ and diagonal $\left(d_{(d) j}, \vec{k}\right)$ ones (equation $3)$. The variances of these coefficients $\left(\sigma_{(h)}, \sigma_{(v)}\right.$ and $\left.\sigma_{(d)}\right)$-equation 2- are independent among them. This implies the definition of three different $\gamma$ values: $\left(\gamma_{(h)}, \gamma_{(v)}, \gamma_{(d)}\right)($ equation 3).

GSA Algorithm . The algorithm consists of the following steps:

1. Evaluate the associated variances to the wavelet coefficients in each of the levels and for each of the bands (horizontal, vertical and diagonal). It is assumed that these values follow a Gaussian distribution centred at cero $(\mu=$ 0 ). The dispersion $\sigma$ at level $\mathrm{j}$ is obtained by using the following equations:

$$
\left(\sigma_{(\cdot) j}\right)^{2}=\operatorname{Var}\left[d_{(\cdot) j}, \vec{k}\right]
$$


2. Adjust the logarithm of the variances, obtained in the previous step, to a straight line[12]:

$$
\log _{2}\left(\operatorname{Var}\left[d_{(\cdot) j,} \vec{k}\right]\right)=-j \gamma_{(\cdot)}+a_{(\cdot)}
$$

We have three kinds of coefficients, so the process has to be repeated for each of them, producing three values of slope: $\left(\gamma_{(h)}, \gamma_{(v)}, \gamma_{(d)}\right)$, and three ordinate values $\left(\left(a_{(h)}, a_{(v)}, a_{(d)}\right)\right.$. As low levels have not sufficient number of coefficient values to consider them statistically significant, we can reject them. In this way, we consider only variances from level 3 and up.

3. Randomly generate the values of the coefficients for the new levels. The generated values follow a Gaussian distribution centred at cero and with dispersion obtained using equation 2. This guarantees the same statistical behavior as for the original levels.

4. Generate the new level of the terrain mesh by calculating the inverse wavelet transform of the coefficients obtained in step 3.

Figure 1 shows a visual example where we have applied this approach has been applied to generate a finer resolution of a natural terrain mesh.

This approach offers good results when the fractal dimension of the original mesh is more or less homogenous across the whole surface (figure 1, top). However, most of the practical cases do not follow the previous conditions. For instance, landscapes including rough mountains and smooth valleys (figure 1 , bottom), have no homogeneous fractal dimension. Figure 1 shows that the results of GSA in this case of heterogeneous terrains is not so good as those obtained where the the homogenty in the fractal dimension is accomplished.

\subsection{Local Scope Approach (LSA)}

As indicated at the end of the next section, the GSA method presents serious deficiencies to its application to general meshes. To solve these problems we have introduced the local scope approach that can be used in the common DEMs that are better represented as multifractal objects.

Even though multifractals have been extensively studied, there are not too many real applications using this theory. A possible cause is the mathematical complexity involved in its use. The last remark is important because our goal is to develop an algorithm efficient enough to dynamically generate new LODs. To accomplish both objectives: good characterization of the fractal nature of DEMs, and good computational performance, we assume the following constraints:

1. The mesh is divided into regions, each of them has a more or less homogeneous fractal dimension.

2. The fractal dimension transition across neighbour regions is smooth.

The first assumption does not impose important constraints because it has been well established in the literature that DEMs present a locally monofractal behavior. In fact, most of the analysis and synthesis algorithms related to terrain 
meshes are based on $\mathrm{fBm}$. The second condition might be more difficult to accomplish in some cases, but its influence in the final result is not so critical; partial violation of it produces still good enough final meshes.

Local Fractal Dimension Estimation. One of the key points in LSA, as it was in GSA, is the estimation of the $\gamma$ parameter. In LSA we have a different $\gamma$ parameter at each location. The $\gamma$ estimation is based on the use of the variance of the detail coefficients of the WT. However, we only consider the coefficients which have a spatial domain close to the location where we are estimating the $\gamma$ value.

Being $X(x, y)$ a function (accomplishing the previously stated conditions)to which we have previously applied the WT, we can conclude that around each point $\vec{r}=(x, y)$ there is a region that satisfies equation 3 with a single value of the $\gamma$ parameter.

As in the GSA, our meshes are discrete functions parametrized by two parameters, then WT produces three different types of detail coefficients: horizontal $\left(d_{(h) j,}, \vec{k}\right)$, vertical $\left(d_{(v) j}, \vec{k}\right)$ and diagonal $\left(d_{(d) j}, \vec{k}\right)$ ones. The variances associated to each type of coefficients are independent among themselves, and can be derived from equation:

$$
\left(\sigma_{(\cdot) j}(\vec{r})\right)^{2}=\operatorname{Var}\left[d_{(\cdot) j,} \vec{k}\right](\vec{r})=A_{(\cdot)}(\vec{r}) 2^{-j \gamma_{(\cdot)}(\vec{r})}
$$

being $A_{(\cdot)}(\vec{r})$ a constant through the different levels $j$, and $\gamma_{(\cdot)}(\vec{r})$ the local $\gamma$ parameter at point $\vec{r}$.

The next step will be able the definition of the spatial regions with homogeneous fractal dimension. This will determine the particular sets of coefficients to be used. To solve it in a accurate way is not trivial. It implies an additional computational cost that will be incompatible with our temporal restrictions. To overcome this limitation we make an important simplification: actual regions are not determined. Instead we suppose that the fractal dimesion is more or less homogeneous in a square window centred at the current point, so only coefficients inside the window are used to estimate the $\gamma_{(\cdot)}(\vec{r})$ parameters.

The previous simplification is not free of risk, because the final result depends on the proper selection of the window size. The selection of the appropiate windows size may require some kind of trial and error test.

Once the window size has been selected, we always consider the same number of coefficients, no matter the level we are working on, to obtain the local variance.

Values $\left(\gamma_{(h)}, \gamma_{(v)}, \gamma_{(d)}\right)$ are calculated as the slope of the straight line represented by the equation:

$$
\log _{2}\left(\operatorname{Var}\left[d_{(\cdot) j, \vec{k}}\right]\right)(\vec{r})=-j \gamma_{(\cdot)}(\vec{r})+a_{(\cdot)}(\vec{r})
$$

Due to the exponential increase of spatial domain covered by detail coefficients when the level decreases, only variance of the coefficients at higher levels is considered. 
New LODs Generation. Once the set of $\gamma_{(\cdot)}(\vec{r})$ and $a_{(\cdot)}(\vec{r})$ values have been evaluated, we are ready to generate the additional levels of detail. To generate the vertex that conforms with the new LOD mesh at level $n$ we use, as in the GSA, the inverse wavelet transform applied to the synthetic coefficients generated randomly. The random generation is based on a Gaussian distribution centred at cero and having its dispersion expressed by the following equation:

$$
\sigma_{(\cdot) n}^{2}(\vec{r})=2^{a_{(\cdot)}(\vec{r})} 2^{-m \gamma_{(\cdot)}}(\vec{r})
$$

The process can be repeated as many times as needed until we achieve the desired resolution in the final terrain mesh. However, a degradation in the quality of the final result has been observed when the number of extra detail levels is too high.

LSA Algortim. As a summary of LSA we will present the specific steps involved in this solution. If we have $m$ initial levels generated using actual data, and if $n$ is the number of extra levels of detail (the final number of levels will be $m+n$ ) the algorithm associated to LSA will repeat the following steps for each point at level $m$ :

1. Evaluate the local variance of the detail coefficients at levels $j<m$ (this process has to be done for the three types of coefficients).

2. Calculate the values of the slopes $\left(\gamma_{(h)}(\vec{r}), \gamma_{(v)}(\vec{r}), \gamma_{(d)}(\vec{r})\right)$ and the ordinate values $\left(a_{(h)}(\vec{r}), a_{(v)}(\vec{r}), a_{(d)}(\vec{r})\right)$, using equation 3 .

3. Generate randomly, using a Gaussian distribution centred at cero and with the dispersion obtained from equations 5 , the new details coefficients for levels from $m$ to $m+n-1$ related to the current point $\vec{r}$.

Once we have applied the previous algorithm to every point at level $m$ we calculate the inverse wavelet transform for the new added coefficients obtaining the extra terrain levels of detail.

\subsection{Results}

To test the presented approaches we have used two different meshes. The first mesh contains only rough mountain areas (figure 1,top), and the second one includes rough mountains and smooth valleys (figure 1, bottom).

The quality of the results will depend on three main parameters: the approach selected (local or global), the selected wavelet base, and, in the case of local scope approach, the window size. To test the last parameter we have selected square windows of four different window sizes: $1 \times 1,3 \times 3,5 \times 5$ and $7 \times 7$.

The estimation of the goodness of the results will be based on two main criteria, related to our original objectives: visual quality and computing performance. The first aspect is somewhat subjective while the second will be based on measuring of the time spent for the new level generation. 
The two original terrain meshes have a size of $16 \times 16$ with 4 actual level of detail (figures 1, left). We have increased the mesh resolution up to $64 \times 64$ points, which implies the generation of two extra levels of detail.

Figure 2 shows the results of applying the LSA, with a linear-spline wavelet base, to the hetereogeneous test mesh. Four different window sizes have being used for the test. Table 1 presents the time to generate the extra levels of detail for both approaches. Time measures have been performed using a Pentium II $233 \mathrm{MHz}$ processor.

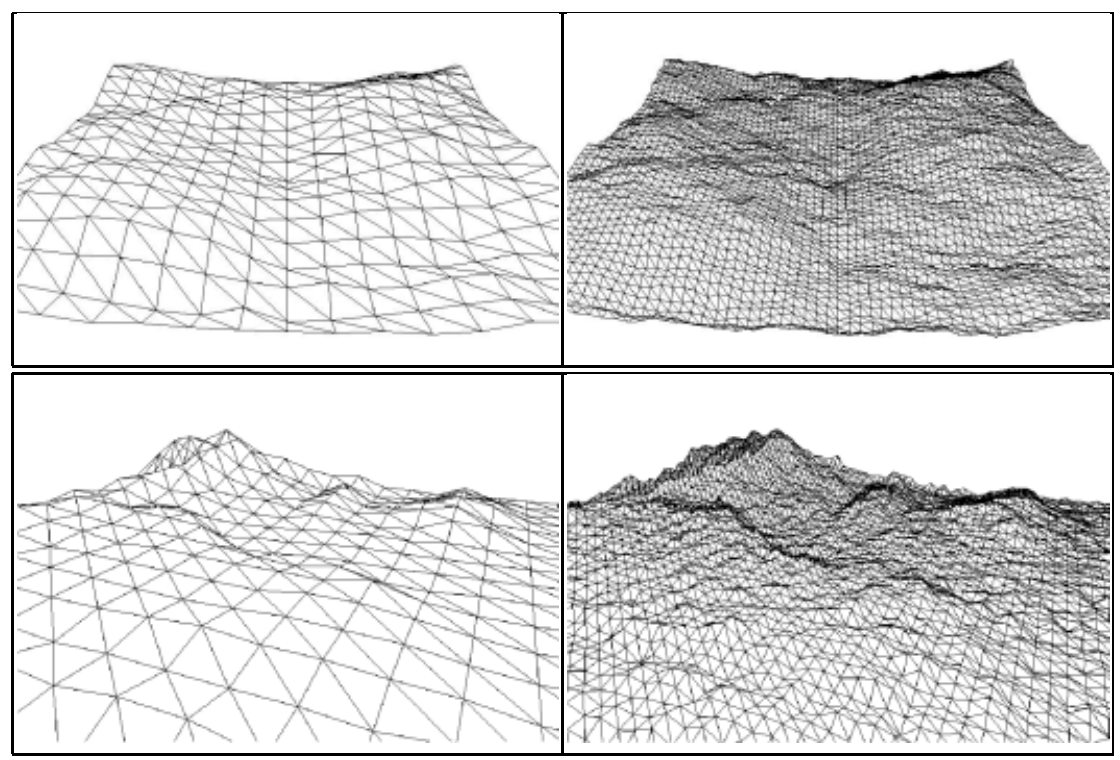

Fig. 1. Top-Left: Original homogeneous mesh, $(16 \times 16)$ points. Top-Right: Results after applying GSA to homogeneous mesh . Synthetic mesh has $(64 \times 64)$ points. BottomLeft: Original hetereogeneous mesh, $(16 \times 16)$ points. Bottom-Right:Results of applying GSA to hetereogeneous mesh. Synthetic mesh has $(64 \times 64)$ points.

Table 1. Spent Time (ms.) for the different approaches (linear-spline wavelet base).

\begin{tabular}{|l|c|c|c|c|c|}
\hline & GSA & LSA 1x1 & LSA 3x3 & LSA 5x5 & LSA 7x7 \\
\hline Homogeneous M. & 78 & 95 & 100 & 122 & 132 \\
\hline Heterogeneous M. & 77 & 95 & 100 & 120 & 130 \\
\hline
\end{tabular}




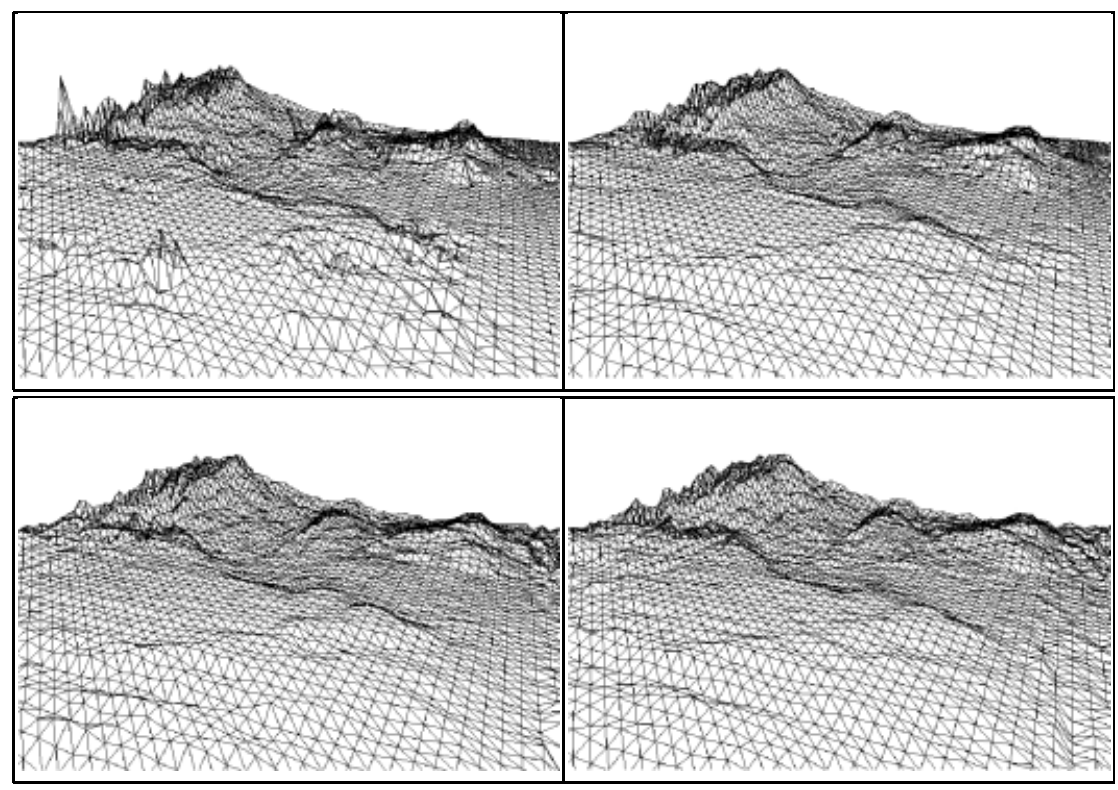

Fig. 2. Results applying LSA to heterogeneous mesh with window size of $: 1 \times 1,3 \times 3$, $5 \times 5$, y $7 \times 7$.

\section{Conclusions and future work}

In this paper, two new methods (GSA and LSA) have been presented to increase actual terrain resolution based on a fractal-wavelet approach. GSA analyses the global statistical properties to decide how to extend the new LODs terrains, while LSA analyses these statistical properties locally at each point. As a first conclusion, we can observe that both methods are fully compatible with our temporal restrictions imposed for real-time terrain representation, allowing a dynamical generation of extra levels of detail on demand.

GSA has been proved to be a good approach when used on terrains with uniform geographical properties(figure 1, top), but it fails in not so much homogeneous terrains (figure 1, bottom). This behavior is not strange because global statistical analysis tends to minimize specific local properties having as a result a uniform distribution for the whole mesh. To overcome this problem, the special analysis used in LSA preserves local properties of terrain using only coefficients close to the particular location (figure 2). The $25 \%$ extra time of this method (table1), considering a $3 \times 3$ window size, is justified taking into account the achieved improvement in the final visual appearance.

As indicated in section three, the vanish moments of the wavelet bases have influence on the achieved degree of proximity to pure fBm. Tests performed using others symetric different wavelet bases have proved that this influence has no very important consequences in the final visual appearance. For cost reasons the recomended base for interactive applications is the linear-spline. 
The window size in LSA is another important aspect for the quality results. Tests indicate that window sizes inferior to $3 \times 3$ have got no enough values to perform reliable statical analysis, this is clearly reflected in the visual appearance. On the contrary, too large window size (superior to $5 \times 5$ ) are not capable reflecting local essence at each point of the surface, becoming in a behavior similar to GSA (figure 2).

As indicated in the introduction of this paper, the other important component in terrain representation is the realistic textures mapped to the terrain mesh. The fractal nature of these terrain textures suggest a possible extension of the presented works to generate finer resolution images from original textures.

\section{References}

1. M. F. Barnsley, R. L. Devaney, B. B. Mandelbrot, H. O. Peitgen, D. Saupe, and R. F. Voss. The Science of fractal images. Springer-Verlag, 1988. ISBN 0387966080 .

2. I. Daubechies. Orthogonal bases of compactly supported wavelets. Commun. Pure Appl. Math, 41:909-996, 1988.

3. C. E. Bolb F. K. Musgrave and R. S. Mace. The synthesis and renderin of eroded fractal terrains. In SIGGRAPH'89, Computer Graphics Proceedings, pages 41-50, 1989.

4. Patrick Flandrin. Wavelet analysis and synthesis of fractional brownian motion. IEEE Transactions on Information Theory, 38(2):910-917, 1992.

5. U. Frisch and G. Parisi. Fully developed turbulence and intermittency. In Int. Summer School on Turbulence and Predictability in GeoPysical Fluid Dynamics and Climate Dynamics, pages 84-88, 1985.

6. S. G. Mallat. A theory for multiresolution signal decomposition: the wavelet representation. IEEE Trans. Patt. Anal. Machine Intell., 11:674-693, 1989.

7. B. B. Mandelbrot. Fractional brownian motion, fractional noises and applications. In SIAM Review 10, pages 422-437, 1968.

8. B. B. Mandelbrot. Intermittent turbulence in self similar cascades: Divergence of high moments and dimensionof the carrier. Fluid Mech., 62(3):331, 1974.

9. B. B. Mandelbrot. The Fractal Geometry of Nature. W. H. Freeman and Co., New York, 1982.

10. B. B. Mandelbrot. Fractals and scaling in Finance. Springer, New York, 1997.

11. B. B. Mandelbrot and C. J. Evertsz. Multifactility of the armonic measure on fractak aggregates and extended self-similarity. Physica, A 177:386-393, 1991.

12. Gregory W. Wornell. Wavelet-based representations for the $1 / \mathrm{f}$ family of fractal procesess. Proceedings of the IEEE, 81(10):1428-1450, 1993.

13. Gregory W. Wornell and Alan V. Oppenheim. Estimation of fractal signals from noisy measurements using wavelets. IEEE Transactions on Signal Prcessing, 40(3):785-800, 1992. 